\title{
Influence of pulsed magnetic field therapy on hand function post flexor tendon repair: a randomized controlled trial
}

Rania Reda Mohamed ${ }^{1}$, Hamada Ahmed Hamada², Eman M. Othman ${ }^{3}$, Abdullah M. Al-Shenqiti ${ }^{4}$, Noha Elserty ${ }^{5}$

\begin{abstract}
${ }^{1}$ Basic Science Department, Faculty of Physical Therapy, Cairo University, Cairo, Egypt 2Biomechanics Department, Faculty of Physical Therapy, Cairo University, Cairo, Egypt ${ }^{3}$ Department for Surgery, Faculty of Physical Therapy, Cairo University, Cairo, Egypt ${ }^{4}$ Faculty of Medical Rehabilitation Sciences, Taibah University, KSA, Saudi Arabia ${ }^{5}$ Basic Science Department, Faculty of Physical Therapy, Benha University, Benha, Egypt
\end{abstract}

Submitted:15 September 2021; Accepted: 19 December 2021

Onine publication: 27 December 2021

Arch Med Sci

DOI: https://doi.org/10.5114/aoms/145137

Copyright @ 2022 Termedia \& Banach

\begin{abstract}
Introduction: The study was conducted to investigate the influence of pulsed magnetic field therapy on hand function, grip and pinch grip strength in post flexor tendon repair patients.

Material and methods: Fifty male patients with flexor tendon repair took part in this research, with ages ranging from 25 to 50 years. They were assigned randomly into two groups: Group A (experimental) received pulsed magnetic therapy together with exercise, whereas Group B (control) received only exercise. Evaluation of hand grip strength with Jamar hydraulic hand dynamometer, pinch strength with baseline pinch gauge and hand function with Michigan Hand Outcomes Questionnaire, all measurements were made before and after the treatment. They received 16 sessions in 8 weeks ( 2 sessions/week). Results: $2 \times 2$ mixed design MANOVA revealed no significant difference between both groups pre- and post-treatment in MHOQ total, function, aesthetic $(p>0.05)$ and in hand grip strength while there was a significant difference across groups after treatment in the strength of pinch grip, MHOQ ADL, pain and satisfaction $(p<0.05)$; there was also a significant difference between pre- and post-treatment within groups in all outcome measures $(p<0.05)$.

Conclusions: PMF increases the efficiency of physical therapy treatment, and it also increases the strength of both hand grip and pinches grip in patients after flexor tendon repair in zone II.
\end{abstract}

Key words: magnetic field, pinch grip, dynamometer, Michigan Questionnaire.

\section{Introduction}

The hand is the most active part of the body, and its basic function is essential for everyday tasks. This typical work depends upon the integrity of its structures including bones, tendons, and neurovascular structures. There are numerous tendons passing and inserted in the hands, including the flexor tendons which are classified according to their characteristics into five zones [1, 2].

Flexor tendon injuries are classified according to these five anatomical zones. Zone I is extending from Flexor Digitorum Profundus that

\author{
Corresponding author: \\ Hamada Ahmed Hamada \\ Biomechanics \\ Department \\ Faculty of \\ Physical Therapy \\ Cairo University, \\ Cairo, Egypt \\ Phone: 00201117893697 \\ E-mail: hamada.ahmed@ \\ pt.cu.edu.eg
}


insert on/that is inserted in the distal phalanx to Flexor Digitorum Superficialis. The space between Flexor Digitorum Superficialis and the nearby boundary of A1 pulleys stands for Zone II. The area that expands between the boundary of the transverse carpal ligament and the A1 pulleys in the palm of the hand to a wrist represents Zone III. The tendons in the carpal tunnel represent Zone IV. Zone $\mathrm{V}$ is the area in the forearm region that extends from the carpal tunnel to the origin of the tendons [3].

Flexor tendon injuries are the most important traumatic wounds that occur in hands particularly in zone II, which is most affected, and it has a great implication on the daily living activities due to its anatomical position. Injury in zone II leads to decreased grip strength due to the ineffective pulley system which incorporates bowstringing, decreased composite flexion, and may lead to stiffness [4]. Surgical repair of injured tendons is the major option for restoring the normal length, strength, and gliding excursion of the tendons. Several factors, such as collateral nerve damage, repair procedure, surgeon expertise, and postoperative recovery, lead to the effectiveness of the surgical repair. Recent studies pointed out to the importance of early proper rehabilitation and early mobilization as a contributing factor for surgical repair success $[5,6]$.

There are several complications that followed the surgical repair of tendons such as decreased hand mobility and function, adhesion formation, muscle atrophy, loss of normal tendon excursion, and loss of muscle power. This gives rise to the importance of physical therapy programme to overcome or prevent these complications from regaining/in order to regain the normal function of the hand [7]. Some research studies applied on animals examined the impact of ultrasound and pulsed magnetic field (PMF) on tendon repair but they yielded controversial results [8-10].

It was postulated that PMF influenced some biological functions as it helps in decreasing pain and inflammation of the musculoskeletal system [11-13], improve wound and bone healing and enhance regeneration of neural tissues [14, 15]. These effects occur due to increased peripheral blood perfusion, which is a sign of the local increase in the oxidation process. The effect of PMF on the healing process depends on better oxygen supply that leads to better energy supply; pulsed magnetic fields are most effective with extremely low frequencies [16].

Although some studies were applied to examine the impact of PMF on different tissues and assessed its implementation in different clinical situations, the effect of PMF remains constrained and needs more clarification concerning its influ- ence on tissues and the application parameters that should be used in different clinical situations [17]. So, our aim was to examine the impact of PMF on hand function, grip and pinch grip strength post repair of the hand flexor tendon in male patients.

\section{Material and methods}

\section{Participants}

Fifty male patients with post flexor tendon repair (repair is Bruner zig-zag and Bunnell incision) were recruited from the hand surgery clinic, El Sahel Teaching Hospital, Cairo, Egypt. Their age was ranging from 25 to 50 years (only male patients were included in this study due to differences in hand grip parameters between both sexes) [18]. All the patients had a repair surgery after damaging dominant hand's flexor tendon caused by cutting wounds in zone II. The participants were excluded if they had secondary repair surgery, tendon injury due to the fingers rapid forceful extension, diabetes mellitus, nerve injury or bone fracture associated with a tendon injury and if they had any complications that occurred during surgery.

\section{Study design}

A prospective, randomized, single-blind, prepost-test, controlled experiment was planned for this study. The Institutional Review Board at Cairo University's Faculty of Physical Therapy provided ethical approval (No. P.T. REC/012/002595) and Pan African Clinical Trial Registry with the number (PACTR202002716709938) before initiating the investigation. The Declaration of Helsinki Guidelines for Human Research were followed when conducting this study. The study took place between April 2020 and December 2020.

\section{Randomization}

Each participant signed an informed written consent form after being informed about the nature, aim, and benefits of the study in addition to the right to decline or withdraw at any time, as well as the secrecy of any information received. Using sealed opaque envelope procedures, subjects were randomly allocated to either Group A (experimental) or Group B (control). A lottery was employed to randomize the sample, and the sealed envelope containing letters (A or B) was used before enrolment in the study. The randomization was constrained to allow blocks to ensure that all groups had an equal number of participants. There was no drop out after randomization as shown in the Figure 1 [1]. 


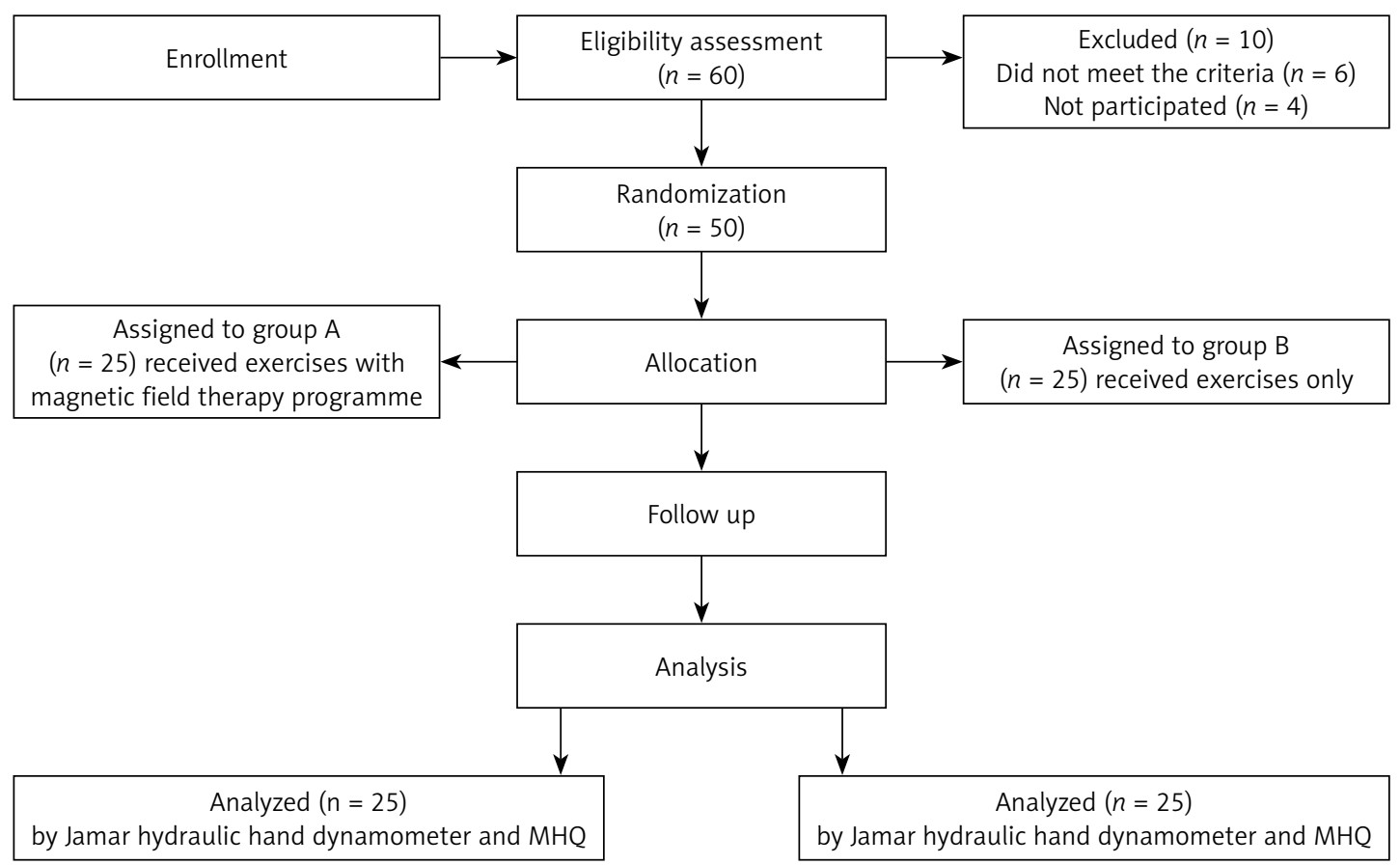

Figure 1. The participants' flow chart

\section{Assessment procedures}

\section{Michigan Hand Outcomes Questionnaire} $(\mathrm{MHQ})$

It has six scales to assess hand function, including general hand function, daily life activities, work performance, pain, aesthetics, and level of satisfaction with hand function [19].

High scores on the pain scale indicate higher discomfort, whereas high scores on the other five measures indicate better hand function. For the hand function, the responses were analysed using a Likert scale ranging from 1 to 5 . The scoring of the scales depends on the summation of the responses of each item of the scales. Each participant was instructed to mark the answer to each question and if he was unable to determine a definite answer, he was asked to indicate the best answer which reflects his level [19].

\section{Jamar hydraulic hand dynamometer}

Grip strength was measured using the Jamar Hydraulic Hand Dynamometer (3-piece kit, Patterson Medical, Warrenville, IL, USA). The Jamar Hydraulic Hand Dynamometer kit includes a baseline pinch gauge unit that was used to measure two-point pinch strength. For routine screening and isometric grip strength measurement, it is a valid, reliable, and effective hand assessment method. Dual-scale reading, a peak-hold needle, and an adjustable handle are part of the dynamometer. For pinch measurement, there is also a pinch gauge unit. The dynamometer has an ad- justable handle for intervals of 1, 1.5, 2, 2.5 and 3 inches spacing. Up to 200 pounds and $90 \mathrm{~kg}$ of the measuring scale used in the dynamometer are defined. The American Society of Hand Therapists recommended that grip and pinch strength be measured following their guidelines. Before utilizing the dynamometer, the adjustable handle is set to correct spacing (typically 1.5 inch spacing for standardization), and the peak hold needle is calibrated to zero. Before measurement, calibration of the dynamometer was done. Procedures for evaluation were applied before and after the rehabilitation programme (after 8 weeks).

Participants were guided to sit on a chair without armrests. Shoulder adduction, neutral rotation, $90^{\circ}$ elbow flexion, mid- forearm position, $30^{\circ}$ wrist flexion and $15^{\circ}$ ulnar deviation were carried out by each participant. To achieve maximum grip strength, participants were asked to push on the handle of the dynamometer as much as possible. To prevent fatigue, measurements were taken three times with 10-20 s' rest in between and the average of the three trials was calculated and reported. Baseline Pinch Gauge: in the same manner, the pinch strength assessment was performed, and participants were grasping the baseline pinch gauge by the pad of the thumb and the pad of the index finger [20,21].

\section{Treatment procedures}

\section{Exercise programme}

The physical therapist performed this exercise programme for all patients. Each patient got 
a passive composite complete fist, a passive distal interphalangeal joint extension, and flexion of the metacarpophalangeal and proximal interphalangeal joints, metacarpophalangeal joint block in full flexion and actively extending the interphalangeal joint, passive distal interphalangeal joint flexion and active extension, metacarpophalangeal joint block in full flexion and actively extending the interphalangeal joint, passive distal interphalangeal joint flexion and active extension, passive proximal interphalangeal joint flexion and active extension, the glide of unaffected fingers with independent flexor digitorum superficialis for 0 3 weeks, hold for isolated flexor digitorum superficialis glide of involved digits was added in week 3 . Active, non-resistive digital flexion and extension were introduced in week 4 . In week 5 , for distal interphalangeal joint, proximal interphalangeal joint flexion, gentle blocking exercises were added. Finally, resistive exercises were added in week 8. These exercises were performed 2 times per week for 8 weeks with $10-15$ repetitions for each exercise and the patients were advised to perform these exercises every $2 \mathrm{~h}$ with $10-15$ repetitions at home as a home programme. (This was the protocol carried out by the Rehabilitation Services Department of The Brigham and Women's Hospital, Inc., 2007).

\section{Magnetic therapy}

Magnetic therapy programme (Physio MG 815-low-frequency magnetic field therapy unit) was applied by the physical therapist. With a frequency of $40 \mathrm{~Hz}$ and an induction time of $2.5 \mathrm{mT}$ for 25 min per session, the unit was adapted for the production of a low-frequency magnetic field with a plate applicator intended for local application, whereby the effect of the magnetic field is localized in the treatment area. Subjects received two sessions per week for 8 weeks. There were no complications during the application.

\section{Statistical analysis}

Due to a lack of previous research on the influence of pulsed magnetic field therapy on the hand grip, pinch grip strength, and function in male post-flexor tendon repair patients, and the difficulty to evaluate the effect size, a pilot study for a group of ten patients was conducted.

For this sample size, the statistical analysis test (2x2 Mixed Design MANOVA) showed a $p$-value of 0.25 , which was used to detect the effect size using the $G^{*}$ power programme ( $G^{*}$ power 3.0.10). Power analysis showed that 50 patients with a detected effect size of 0.33 were enough to achieve a power level of $95 \%$. With the fear of losing patients during the 8-week study duration, we examined 60 patients during the whole study period.
The Statistical Package for Social Sciences (SPSS) software version 23 for Windows was used for all statistical analyses. Covariance homogeneity and data normality are tested using the Box's test and the Shapiro-Wilk test, respectively. $2 \times 2$ mixed design MANOVA was used to compare the tested variables of interest in different test groups and measurement times. The $\alpha$ level was set at 0.05.

\section{Results}

Mixed design MANOVA revealed that there were significant within-subject effect and treatment*time effect $(F=376.901, p=0.0001$, Partial Eta Squared $=0.987)\left(F=6.205, p=0.0001^{*}\right.$, Partial Eta Squared $=0.548$ ) respectively. Also, there was significant between-subject effect $(F=$ 4.71, $p=0.0001^{*}$, Partial Eta Squared $=0.479$ ). The descriptive statistics of within and between groups differences at $95 \% \mathrm{Cl}$ for the effects of in terventions for all dependent variables were presented in Table I. Concerning the within-subject effect, the multiple pairwise comparison test was used to compare between pre- and post-treatment in both groups, and it revealed that there was significant increase $(p<0.05)$ in MHOQ total, $A D L$, satisfaction, function, aesthetic, pinch grip and hand grip strength and significant reduction $(p<0.05)$ in MHOQ pain at both groups post-treatment. Regarding between-subject effects, multiple pairwise comparisons revealed that there was no significant difference between both groups pre-treatment and post- treatment in MHOQ total, function, aesthetic and in hand grip strength while there was a significant increase $(p<0.05)$ in pinch grip strength, MHOQ ADL, and satisfaction and a significant reduction $(p<0.05)$ in MHOQ pain post-treatment in favour to group compared to group $B$.

\section{Discussion}

Zone II flexor tendon injuries are one of the most prevalent injuries in the hand [6]. For such an injury, an effective rehabilitation programme and precise surgical intervention are required. The anatomical position of this zone increases the responsibility for tendon adhesion formation because it is an important rehabilitation area. The achievement of good function of the hand is difficult to occur following injuries and repair of the flexor tendon in zone II $[22,23]$. The purpose of this study was to investigate the effectiveness of Pulsed Magnetic Field therapy on the hand grip, pinch grasp, pain, aesthetics, activities of daily living, function, and satisfaction after a flexor tendon repair in zone II.

In the current study, all outcome indicators within each group showed a significant difference before and after treatment. These results indicat- 
Table I. Descriptive and inferential statistics of the dependent variables in the experimental and control groups pre and post the 8-week study period

\begin{tabular}{|c|c|c|c|}
\hline Parameter & $\begin{array}{c}\text { Group (A) } \\
(n=30)\end{array}$ & $\begin{array}{c}\text { Group (B) } \\
(n=30)\end{array}$ & $P$-value* \\
\hline \multicolumn{4}{|l|}{ MHOQ total: } \\
\hline Pre training & $84.08 \pm 4.61$ & $83.6 \pm 5.29$ & $0.734^{\mathrm{NS}}$ \\
\hline Post training & $93.4 \pm 3.01$ & $92.2 \pm 2.59$ & $0.138^{\mathrm{NS}}$ \\
\hline$\%$ of change & $11.08 \uparrow \uparrow$ & $10.28 \uparrow \uparrow$ & \\
\hline$P$-value ${ }^{\star *}$ & $0.001^{\mathrm{s}}$ & $0.001^{\mathrm{s}}$ & \\
\hline \multicolumn{4}{|l|}{ MHOQ Function: } \\
\hline Pre training & $88.8 \pm 2.29$ & $88.16 \pm 3.51$ & $0.45^{\mathrm{NS}}$ \\
\hline Post training & $94.36 \pm 1.49$ & $93.84 \pm 2.86$ & $0.343^{\mathrm{NS}}$ \\
\hline$\%$ of change & $6.26 \uparrow \uparrow$ & $6.44 \uparrow \uparrow$ & \\
\hline$P$-value ${ }^{\star *}$ & $0.001^{\mathrm{s}}$ & $0.001^{\mathrm{s}}$ & \\
\hline \multicolumn{4}{|l|}{ MHOQ ADL: } \\
\hline Pre training & $89.44 \pm 2.5$ & $88.12 \pm 2.86$ & $0.089^{\mathrm{NS}}$ \\
\hline Post training & $96.16 \pm 1.28$ & $94.24 \pm 2.69$ & $0.002^{\mathrm{s}}$ \\
\hline$\%$ of change & $7.51 \uparrow \uparrow$ & $6.94 \uparrow \uparrow$ & \\
\hline$P$-value** & $0.001^{\mathrm{s}}$ & $0.001^{\mathrm{s}}$ & \\
\hline \multicolumn{4}{|l|}{ MHOQ Pain: } \\
\hline Pre training & $7.4 \pm 0.81$ & $7.4 \pm 0.86$ & $0.99^{\mathrm{NS}}$ \\
\hline Post training & $3.4 \pm 0.57$ & $4.2 \pm 0.7$ & $0.001^{\mathrm{s}}$ \\
\hline$\%$ of change & $54.05 \downarrow \downarrow$ & $43.24 \downarrow \downarrow$ & \\
\hline$P$-value ${ }^{\star *}$ & $0.001^{\mathrm{s}}$ & $0.001^{\mathrm{s}}$ & \\
\hline \multicolumn{4}{|c|}{ MHOQ Aesthetics: } \\
\hline Pre training & $83 \pm 1.75$ & $83.56 \pm 2.39$ & $0.351^{\mathrm{NS}}$ \\
\hline Post training & $90.84 \pm 1.7$ & $90.56 \pm 1.93$ & $0.59^{\mathrm{NS}}$ \\
\hline$\%$ of change & $9.44 \uparrow \uparrow$ & $8.37 \uparrow \uparrow$ & \\
\hline$P$-value** & $0.001^{\mathrm{s}}$ & $0.001^{\mathrm{s}}$ & \\
\hline \multicolumn{4}{|c|}{ MHOQ Satisfaction: } \\
\hline Pre training & $85.7 \pm 2.15$ & $85.48 \pm 2.14$ & $0.694^{\mathrm{NS}}$ \\
\hline Post training & $95.08 \pm 1.97$ & $91.68 \pm 2.59$ & $0.001^{\mathrm{s}}$ \\
\hline$\%$ of change & $10.94 \uparrow \uparrow$ & $7.25 \uparrow \uparrow$ & \\
\hline$P$-value ${ }^{\star *}$ & $0.001^{\mathrm{s}}$ & $0.001^{\mathrm{s}}$ & \\
\hline \multicolumn{4}{|l|}{ Pinch grasp: } \\
\hline Pre training & $10.91 \pm 1.67$ & $10.74 \pm 0.93$ & $0.569^{\mathrm{NS}}$ \\
\hline Post training & $16.57 \pm 1.67$ & $15.02 \pm 1.11$ & $0.001^{\mathrm{NS}}$ \\
\hline$\%$ of change & $51.87 \uparrow \uparrow$ & $39.85 \uparrow \uparrow$ & \\
\hline$P$-value ${ }^{\star *}$ & $0.001^{\mathrm{s}}$ & $0.001^{\mathrm{s}}$ & \\
\hline \multicolumn{4}{|c|}{ Hand grip strength: } \\
\hline Pre training & $57.02 \pm 12.39$ & $56.96 \pm 11.83$ & $0.986^{\mathrm{NS}}$ \\
\hline Post training & $96.32 \pm 20.77$ & $92.04 \pm 18.14$ & $0.442^{\mathrm{NS}}$ \\
\hline$\%$ of change & $68.92 \uparrow \uparrow$ & $61.58 \uparrow \uparrow$ & \\
\hline$P$-value ${ }^{\star \star}$ & $0.0001^{s}$ & $0.0001^{\mathrm{s}}$ & \\
\hline
\end{tabular}

*Inter-group comparison; ${ }^{* *}$ intra-group comparison of the results pre and post training. ${ }^{N S} P>0.05-$ non-significant, ${ }^{S} p<0.05-$ significant, $P$ - probability. 
ed that both the exercise programme and PMF are effective methods in rehabilitation after the repair of the hand flexor tendon. This is explained by the fact that early controlled motion in the specified exercise programmes can aid in the reduction of tendon adhesions and joint contractures, which are common problems following surgery [22].

Physical therapy following flexor tendon repair is beneficial in preventing issues such as limiting hand mobility, adhesion development, muscle atrophy, maintaining tendon excursion, muscle strengthening, and preserving hand functionality to regain the normal function of the hand as it was previously [24]. After flexor tendon repair, passive mobilization (exercises according to the Duran Protocol) aids tendon gliding, which decreases or prevents adhesion formation, especially in zone II of injury [25-27].

These exercises are critical in recovering longterm finger dexterity and hand function [28]. Furthermore, research suggests that regulated stress on the tendons, such as that caused by passive or dynamic movement, aids healing, controls early collagen deposition, and promotes biochemical activities that increase tensile strength $[29,30]$.

Also, PMF, which is safe, easily applied and non-invasive modality may be contributed to the difference between pre- and post- treatment in the study group and disparity in post-treatment outcomes between both groups in pinch grip strength, MHOQ ADL, pain and satisfaction as it may enhance the healing mechanism of the body [31].

PMF accelerates the healing process in all phases of repair that occurred directly at the cellular level and do not depend on the anatomical contrasts within the repairing tendon. It was stated that the effectiveness of PMF occurred in the stages of the tendon injury including inflammatory, angiogenesis, and cell proliferation stages [32, 33].

PMF transduction is linked to ion binding in regulatory pathways involving growth factor release, and these growth factors and cytokines are involved in $\mathrm{Ca} /$ calmodulin-dependent tissue development and repair [34-36]. The increased speed and efficiency of the cellular response to primary injury will be beneficial for the healing process as pulsed electromagnetic fields expedite the binding of $\mathrm{Ca} 2$ to calmodulin. It was suggested that PMF enhances the rate of healing through affecting the ion allocation on cell membrane that leads to accelerate the re-establishment of normal potentials [37].

Through the process of magnetohydrodynamics, it also improves vascular supply to the tissue and increases energy consumption and turnover with an increase in ATP [38]. It was suggested that PMF follows the same explanation offered by Melzack and Wall in inhibiting pain through stimula- tion of A delta fibres that produce an inhibitory effect on $C$ fibre that carries the nociceptive stimulus. Also, it was suggested that PMF might inhibit pain by interfering with calcium ion movements, levels of endorphin, the firing of neurons, and producing acupuncture-like action [39, 40].

PMF also affects the voltage-gated calcium and sodium channels that accumulate at the axonal damage or segmental demyelination site, causing cell repolarization at the membrane level [41]. Pulsed magnetic fields were also indicated to reduce joint swelling, improve healing, and improve pain management by reducing the need for pharmaceuticals such as non-steroidal anti-inflammatory drugs. By lessening inflammatory cytokines, it also activates chondrocytes, osteoblasts, and tenocytes. Inflammation is decreased by pulsed electromagnetic fields via the agonist effect on A2A adenosine receptors that reduce interleukin-1 $\beta$, pro-inflammatory cytokines (interleukin- 6 and interleukin-8), tumour necrosis factor- $\alpha$ and tumour necrosis factor- $\beta$ within the synovial fluid [42, 43].

According to the study done on Achilles tendinopathy (AT), it is a medical condition characterized by discomfort, oedema, and impaired performance. It is associated with excessive use, but it is also defined by inflammatory events. Tendon repair is often slow and inadequate, with a higher incidence of degenerative events and a poor response to therapy. The discovery of new and effective conservative techniques to promote tissue repair as adjuvants to surgical intervention is currently being funded. Biophysical stimulations with pulse magnetic fields (PMF) are a non-invasive, cost-effective, and safe conservative treatment for delayed union and non-union fractures that has already been approved by the Food and Drug Administration [44].

According to Kamel et al., to treat symptoms of Carpal Tunnel Syndrome (CTS), two groups underwent nerve and tendon gliding activities that are promoting axonal transport and nerve transmission. The research found that these workouts prevent adhesion formation even while the wrist is immobilized, reduce pressure in the carpal tunnel, and increase the relative excursion of the median nerve and flexor tendons. These advantages were found to be consistent with the findings of the current investigation [45].

In comparison with standard culture conditions, PMF was more successful in boosting C2C12 myogenesis under inflammatory conditions. It can also be used to treat inflammation-related symptoms including muscle atrophy and degeneration [46]. So, using PMF may effectively improve the wound healing process that may enhance the recovery of patients with the repaired tendons. De- 
spite the fact that the current findings revealed no significant differences in specific outcome measures between the two groups, it has a significant impact on pain, $A D L$, and pinch grip strength.

The study was limited by the effectiveness of PMF on each patient's work performance, which was discovered as an item in the MHOQ questionnaire but could not be detected due to socioeconomic issues that forced most of the patients to work despite their hand injury because they were all manual workers, and it must take into account the nature of manual work that may contribute to early return to work during the rehabilitation process. Also, the study was done on one sex and on the dominant-hand injured patient only due to gender and dominance-related differences in the power of grip strength. Further studies may be performed on different types of manual workers to compare between them in assessing the outcome of hand injuries. Also, further studies may be done on different zones rather than zone II on both sexes and both dominant and non-dominant hands.

In conclusion, PMF increases the efficiency of physical therapy treatment as it decreases pain sensation, reduces inflammation, decreases formation of tendon adhesions, enhances the healing process, enhances the strength of hand grip and pinch grip, improves the hand function, satisfaction and aesthetics after repair of the hand flexor tendon in zone II.

\section{Conflict of interest}

This authors declare no conflict of interest.

\section{References}

1. Chase RA. Anatomy and kinesiology of the hand. In: Rehabilitation of the Hand and Upper Extremity. $5^{\text {th }}$ ed. Hunter JM (ed.) Mosby, St Louis 2002; 60-76.

2. Pillip E, Wright II. Flexor and extensor tendon injuries. In: Campbell's Operative Orthopaedics. $11^{\text {th }}$ edn. Canale ST, Beaty JH. Mosby Inc. 2008; 3851-76.

3. De Jong JP, Nguyen JT, Sonnema AJM, Nguyen EC, Amadio PC, Moran SL. The incidence of acute traumatic tendon injuries in the hand and wrist: a 10-year population-based study. Clin Orthop Surg 2014; 6: 196-202.

4. Lilly SI, Messer TM. Complications after treatment of flexor tendon injuries. J Am Acad Orthop Surg 2006; 14: 387-96.

5. Libberecht K, Lafaire C, Van Hee R. Evaluation and functional assessment of flexor tendon 'repair in the hand. Acta Chir Belg 2006; 106: 560-5.

6. Saini N, Kundani V, Patni P, Gupta S. Outcome of early active mobilization after flexor tendons repair in zones 2-5. Indian J Ortop 2010; 44: 314-21.

7. Clark GL. Hand Rehabilitation: A Practical Guide. $2^{\text {nd }}$ ed. Churchill Livingstone, New York, Edinburgh, 1997; 103.

8. Greenough CG. The effect of pulsed electromagnetic fields on flexor tendon healing in the rabbit. J Hand Surg 1996; 21: 808-12.
9. Robotti E, Zimbler AG, Kenna D, Grossman JA. The effect of pulsed electromagnetic fields on flexor tendon healing in chickens. J Hand Surg 1999; 24: 56-8.

10. Maiti SK, Kumar N, Singh GR, Hoque M, Singh R. Ultrasound therapy in tendinous injury healing in goats. J Vet Med A Physiol Pathol Clin Med 2006; 53: 249-58.

11. Lee EW, Maffulli N, Chan KM. Pulsed magnetic and electromagnetic fields in experimental Achilles tendonitis in the rat: a prospective randomized study. Arch Phys Med Rehabil 1997; 78: 399-404.

12. Nicolakis P, Kollmitzer J, Crevenna R, Bittner C, Erdogmus $C B$, Nicolakis J. Pulsed magnetic field therapy for osteoarthritis of the knee a double-blind sham-controlled trial. Wien Klin Wochenschr 2002; 114: 678-84.

13. Winemiller MH, Billow RG, Laskowski ER, Harmsen WS. Effect of magnetic versus sham-magnetic insoles on plantar heel pain. A randomized controlled trial. JAMA 2003; 290: 1474-8.

14. Sweeney KB, Merrick MA, Swez JA. Therapeutic magnets do not affect tissue temperatures. J Athl Train 2001; 36: 27-31.

15. Krylov OA, Antonov AB, Eliseeva ZV, Malikova SN, Shevelev IN. Structural and functional characteristics of recovery of the severed sciatic nerve exposed to pulsed magnetic field. Patol Fiziol Eksp Ther 1993; 4: 29-33.

16. Jacobson J, Gorman R, Yamanashi W, Saxena B, Clayton L. Low amplitude extremely low frequency magnetic field for the treatment of osteoarthritic knees: a double blind clinical study. Altern Ther Health Med 2001; 7: 54-69.

17. Markov M, Colbert A. Magnetic and electromagnetic field therapy. I Back Musculoskelet Rehabil 2000; 15: 17-29.

18. Trish GS, Joseph B, Chad H, Mike C, Kent JA, Mark D. Hand grip strength and gender: allometric normalization in older adults and implications for the NIOSH lifting equation. J Lifestyle Med 2017; 7: 63-8.

19. Kevin CC, Matthew SP, Madonna RW, Rodney AH, Ann A. Reliability and validity testing of the Michigan Hand Outcomes Questionnaire. J Hand Surg Am 1998; 23: 575-87.

20. Fess EE. The effects of Jamar dynamometer handle position and test protocol on normal grip strength. J Hand Surg 1981; 7: 308-9.

21. Fess EE, Moran C. Clinical Assessment Recommendations. American Society of Hand Therapists Monograph, Indianapolis 1981.

22. Dovelle S, Heeter PK. The Washington Regimen: rehabilitation of the hand following flexor tendon injuries. Phys Ther 1989; 69: 1034-40.

23. Evans RB. Managing the injured tendon: current concepts. J Hand Therapy 2012; 25: 173-89.

24. Clark GL. Hand Rehabilitation: A Practical Guide. 2nd ed. Churchill Livingstone, New York, Edinburgh 1997: 103.

25. Spiguel L, Song DH. Grabb and Smith's Plastic Surgery. $6^{\text {th }}$ ed. Lippincott Williams \& Wilkins, Philadephia 2007; 803-9.

26. Libberercht K, Lafaire C, Van Hee R. Evaluation and functional assessment of flexor tendon repair in the hand. Acta Chir Belg 2006; 106: 560-5.

27. Boyer MI, Strickland JW, Engles DR, et al. Flexor tendon repair and rehabilitation: state of the art in 2002. J Bone Joint Surgery 2002; 84: 1684-706.

28. Pettengill KM, van Strien G. Postoperative management of flexor tendon injuries. In: Rehabilitation of the Hand and Upper Extremity. $6^{\text {th }}$ ed. Skirven TM, Terri M (eds.). Elsevier Mosby, Philadelphia 2011. 
29. Buckwalter JA, Grodzinsky AJ. Loading of healing bone, fibrous tissue and muscle: implications for orthopaedic practice. J Am Acad Orthop Surg 1999; 7: 291-9.

30. Sarah G, Ewald OT. A brief historical overview of published postoperative treatment regimens for zone II flexor tendon repairs. Swiss Hand Ther Soc J 2002; 13.

31. Strauch B, Mitesh KP, Daniel JR, Soham MBA, Nelia BBA, Arthur A. Pulsed magnetic field therapy increases tensile strength in a rat Achilles' tendon repair model. J Hand Surg 2006; 31: 1131-5.

32. Aaron RK, Ciombor DK, Simon BJ. Treatment of nonunions with electric and electromagnetic fields. Clin Orthop Relat Res 2004; 419: 21-9.

33. Aaron RK, Boyan BD, Ciombor D, Schwartz Z, Simon BJ. Stimulation of growth factor synthesis by electric and electromagnetic fields. Clin Orthop Relat Res 2004; 419: 30-7.

34. Brighton CT, Wang W, Seldes R, Zhang G, Pollack SR. Signal transduction in electrically stimulated bone cells. J Bone Joint Surg 2001; 83: 1514-23.

35. Pilla AA. Mechanisms and therapeutic applications of time varying and static magnetic fields. In: Handbook of Biological Effects of Electromagnetic Fields. $3^{\text {rd }}$ ed. Barnes F, Greenebaum B (eds.). CRC Press, Palm Beach, FL 2006.

36. Markov MS, Pilla AA. Weak static magnetic field modulation of myosin phosphorylation in a cell-free preparation: calcium dependence. Bioelectrochem Bioenergetics 1997; 233-8.

37. Kokoschinegg P, Fisher G. Effect of pulsed magnetic fields of low intensity on biological system and basic research on this phenomenon. Magnets in Your Future 1992; 6: 5-13.

38. Keltner J. Magnetohydrodynamics of blood flow. Mag Res Med 1990; 16: 139-49.

39. Lee B, Kim C, Lim J, et al. Efficacy of pulsed electromagnetic therapy for chronic lower back pain: a randomized, double-blind, placebo-controlled study. J Int Med Res 2006; 34: 160-7.

40. Thamsborg G, Florescu A, Oturai P, Fallentin E, Tritsaris K, Dissing S. Treatment of knee osteoarthritis with pulsed electromagnetic fields: a randomized, double blind, placebo-controlled study. Osteoarthritis Cartilage 2005; 13: 575-81.

41. Ochoa JL, Campero M, Serra J, Bostock H. Hyperexcitable polymodal and insensitive nociceptors in painful human neuropathy. Muscle Nerve 2005; 32: 459-72.

42. Vincenzi F, Targa M, Corciulo C, et al. Pulsed electromagnetic fields increased the anti-inflammatory effect of $\mathrm{A} 2 \mathrm{~A}$ and $\mathrm{A} 3$ adenosine receptors in human T/C-28a2 chondrocytes and FOB 1.19 osteoblasts. PLoS One 2013; 8: e 65561.

43. Denaro V, Ruzzini L, Barnaba SA, et al. Effect of pulsed electromagnetic fields on human tenocyte cultures from supraspinatus and quadriceps tendons. Am J Phys Med Rehabil 2011; 90: 119-27.

44. Perucca Orfei C, Lovati AB, Lugano G, et al. Pulsed electromagnetic fields improve the healing process of Achilles tendinopathy: a pilot study in a rat model. Bone Joint Res 2020; 9: 613-22.

45. Kamel DM, Hamed NS, Raoof NA, Tantawy SA. Pulsed magnetic field versus ultrasound in the treatment of postnatal carpal tunnel syndrome: a randomized controlled trial in the women of an Egyptian population. J Adv Res 2017; 8: 45-53.

46. Liu M, Lee C, Laron D, et al. Role of pulsed electromag netic fields (PEMF) on tenocytes and myoblasts potential application for treating rotator cuff tears. J Orthop Res 2017; 35: 956-64. 